\title{
Stearic acids at $s n-1,3$ positions of TAG are more efficient at limiting fat deposition than palmitic and oleic acids in C57BL/6 mice
}

\author{
Shiou-Wah Gouk, Sit-Foon Cheng*, Augustine Soon-Hock Ong and Cheng-Hock Chuah \\ Unit of Research on Lipids, Department of Chemistry, Faculty of Science, University of Malaya, \\ 50603 Kuala Lumpur, Malaysia \\ (Submitted 6 June 2013 - Final revision received 7 October 2013 - Accepted 9 October 2013 - First published online 29 November 2013)
}

\begin{abstract}
In the present study, we investigated the effect of long-acyl chain SFA, namely palmitic acid (16:0) and stearic acid (18:0), at $s n-1,3$ positions of TAG on obesity. Throughout the 15 weeks of the experimental period, C57BL/ 6 mice were fed diets fortified with cocoa butter, sal stearin (SAL), palm mid fraction (PMF) and high-oleic sunflower oil (HOS). The $s n-1,3$ positions were varied by 16:0, 18:0 and 18:1, whilst the $s n$-2 position was preserved with $18: 1$. The HOS-enriched diet was found to lead to the highest fat deposition. This was in accordance with our previous postulation. Upon normalisation of total fat deposited with food intake to obtain the fat:feed ratio, interestingly, mice fed the SAL-enriched diet exhibited significantly lower visceral fat/feed and total fat/feed compared with those fed the PMF-enriched diet, despite their similarity in SFA-unsaturated fatty acid-SFA profile. That long-chain SFA at $s n-1,3$ positions concomitantly with an unsaturated FA at the $s n$-2 position exert an obesity-reducing effect was further validated. The present study is the first of its kind to demonstrate that SFA of different chain lengths at $s n-1,3$ positions exert profound effects on fat accretion.
\end{abstract}

Key words: Positional fatty acids: sn-1, 3 positions: SFA: Stearic acid: Fat deposition: Obesity

Obesity is a cardiovascular risk factor that is also associated with a substantial increase in the risk of type 2 diabetes, hypertension and lipoprotein metabolism disorders. Concerted efforts have been made to establish the relationship between obesity and lipid intake; yet, most of the studies are merely based on the total $\mathrm{SFA}^{(1-4)}$, total unsaturated fatty acid ${ }^{(1-5)}$, MUFA $^{(3)}, n-6$ PUFA $^{(3,6-9)}$ and n-3 PUFA ${ }^{(8,10-12)}$ contents of edible oils. In general, a high-SFA-rich diet is perceived to be more obesogenic than diets with lower saturated contents ${ }^{(1-3)}$. However, it has been reported that cocoa butter (COB), a typical saturated fat that contains $63 \mathrm{~g}$ SFA/100 g total fatty acids, causes less weight gain when compared with safflower and olive oils ${ }^{(4)}$. A study carried out by Matsuo et al. ${ }^{(3)}$ has shown positive correlations of carcass fat content with palmitic, stearic and oleic acids and a negative correlation with linoleic acid in rats. Thus, an excessive intake of MUFA is also postulated to exert detrimental effects on adiposity ${ }^{(3)}$.

There has been some controversy regarding the effect of n-6 PUFA, in particular, linoleic acid, on obesity. In recent studies, linoleic acid has been reported to exert pro-obesity effects using rodents ${ }^{(5,6)}$; notwithstanding this, some researchers have postulated $n-6$ PUFA to be most effective at limiting fat accretion ${ }^{(3,7,8)}$. A recent review article has disclosed that the role of $n-6$ PUFA in obesity remains vague as no conclusive inference can be drawn from the available epidemiological evidence $^{(9)}$. Additionally, $n$-3 PUFA ${ }^{(10,11)}$ have also been claimed to reduce fat deposition; yet, no definite results could be obtained by a systematic review evaluating the effect of $n$-3 PUFA on body-mass gain ${ }^{(12)}$. It is evident that the reported results contradict each other. Moreover, there is a paucity of information regarding the positional fatty acids of TAG and their associated effects on obesity.

Kojima et $a l^{(13)}$ reported that dietary 1(3)-behenoyl-2, 3(1)-dioleoylglycerol, which contains long-chain saturated behenic acid $(22: 0)$ at $s n-1$ or $s n-3$ position, prevents visceral fat deposition. Our recent in vivo study has also revealed that long-chain SFA tend to reduce fat deposition if present at $s n-1$ and $s n-3$ positions ${ }^{(14)}$. An exceptional direct negative correlation has been found between fat/feed and total SFA content at $s n-1,3$ positions ${ }^{(14)}$. If long-chain SFA are present at $s n-1,3$ positions, they might not be readily absorbed upon hydrolysis due to the formation of Ca soaps and consequently enter the distal gut and colon, where they are excreted ${ }^{(15,16)}$.

Prior literature has disclosed that the excretion of SFA is also directly related to their chain lengths ${ }^{(13)}$. Henceforth, we hypothesised that further alleviation of fat deposition might

Abbreviations: COB, cocoa butter; HOS, high-oleic sunflower oil; PMF, palm mid fraction; POSt, 1-palmitoyl-2-oleoyl-3-stearoyl glycerol; SAL, sal stearin; StOSt, 1,3-distearoyl-2-oleoylglycerol.

*Corresponding author: Dr S.-F. Cheng, fax +603 7967 4193, email sfcheng@um.edu.my 
occur if stearic acids rather than palmitic and oleic acids are mainly present at $s n-1,3$ positions. In the present study, the effect of common long-chain SFA, namely palmitic acid (16:0) and stearic acid (18:0), at $s n-1,3$ positions of TAG on obesity was investigated using COB, sal stearin (SAL), palm mid fraction (PMF) and high-oleic sunflower oil (HOS). Feed efficiency in terms of fat deposition, i.e. fat/ feed, was determined to accurately interpret the diet-induced adipogenic effects of the test oils.

\section{Materials and methods \\ Diet}

Autoclavable standard pellet feeds (digestible energy $14.0 \mathrm{MJ} / \mathrm{kg}$ ) were purchased from Specialty Feeds. The nutrient composition of the autoclavable standard pellet feeds is given in the Supplementary material (available online). Refined, bleached and deodorised SAL, also known as Shorea robusta, was a gift from Mewah Oil Sdn. Bhd. Refined, bleached and deodorised COB, PMF and HOS were obtained from Intercontinental Specialty Fats Sdn. Bhd. The total and positional fatty acid compositions of dietary oils are given in Tables 1 and 2 , respectively. The diets were prepared by fortifying the standard pellet feeds with the test oils at a composition of $150 \mathrm{~g} / \mathrm{kg}$ diet; subsequently, the mixtures were pelleted into original shape. These diets were left to dry at $30^{\circ} \mathrm{C}$ overnight before being fed to the mice. All the test diets were isoenergetic ( $20 \mathrm{MJ} / \mathrm{kg}$; $60 \%$ of energy from fat).

\section{Animals}

A total of forty weaned male $\mathrm{C} 57 \mathrm{BL} / 6$ mice were obtained from Monash University. The mice were acclimatised to the environmentally controlled laboratory environment $\left(23 \pm 2^{\circ} \mathrm{C}\right.$ and a relative humidity of $60 \%$ ) under a $12 \mathrm{~h}$ light $-12 \mathrm{~h}$ dark cycle. Subsequently, they were randomly divided into four

Table 1. Total fatty acid compositions of dietary oils and fats $(\mathrm{g} / 100 \mathrm{~g}$ of total fatty acids)

\begin{tabular}{ccccc}
\hline & \multicolumn{4}{c}{ Total fatty acid composition } \\
\cline { 2 - 5 } Acyl chain & COB & SAL & PMF & HOS \\
\hline $12: 0$ & ND & ND & 0.05 & ND \\
$14: 0$ & $0 \cdot 10$ & 0.02 & 0.84 & 0.04 \\
$16: 0$ & 25.55 & 4.55 & 58.24 & 3.84 \\
$18: 0$ & 36.61 & 51.83 & 5.46 & $2 \cdot 88$ \\
$20: 0$ & 1.17 & 8.00 & 0.37 & ND \\
$22: 0$ & ND & 0.50 & ND & ND \\
Total SFA & 63.43 & 64.90 & 64.96 & 6.76 \\
$16: 1 n-7$ & 0.23 & ND & 0.06 & 0.10 \\
$18: 1 n-9$ & 33.48 & 33.91 & 31.93 & 82.42 \\
$20: 1 n-9$ & 0.05 & 0.08 & 0.05 & 0.26 \\
Total MUFA & 33.76 & 33.99 & 32.04 & 82.78 \\
$18: 2 n-6$ & 2.62 & 0.95 & 2.96 & 10.37 \\
$18: 3 n-3$ & 0.17 & 0.14 & 0.04 & 0.09 \\
$20: 2 n-6$ & 0.02 & 0.02 & ND & ND \\
Total PUFA & 2.81 & 1.11 & 3.00 & 10.46 \\
\hline
\end{tabular}

$\mathrm{COB}$, cocoa butter; SAL, sal stearin; PMF, palm mid fraction; HOS, high-oleic sunflower oil; ND, not detected. groups of ten mice each and housed individually. The average body masses of the mice did not differ significantly after randomisation $(P=0.089)$ and were 19.7 (SD 0.9), 19.9 (SD 1.2), 20.3 (SD 0.7) and $19 \cdot 7$ (SD 1.2) g for the COB, SAL, PMF and HOS dietary groups, respectively. Throughout the 15 weeks of the experimental period, the mice were given ad libitum access to water and food. Food intake was determined on a daily basis, whereas the body mass of the mice was recorded weekly. The actual food intake was corrected for spilled food after separating it from the faeces of the mice. Fresh faecal samples were collected daily and stored at $-20^{\circ} \mathrm{C}$. The mice were killed by dislocation of the neck. The subcutaneous and visceral adipose tissues were removed from the carcasses, weighed and kept frozen at $-80^{\circ} \mathrm{C}$. The relative amount of total fat is expressed as the fat mass percentage in mouse carcass. Data on body-mass gain and mass of fat deposited were subjected to normalisation with total food intake, shorthanded as gain/feed and fat/feed, respectively. All the protocols followed in the in vivo study were approved by the Animal Care and Use Committee, University of Malaya, Kuala Lumpur, Malaysia (ethic no: KIM/22/11/ 2011/GSW (R)).

\section{Lipid analysis}

The extraction and isolation of lipids from adipose tissue and faecal samples were carried out in accordance with a modified $\operatorname{method}^{(17)}$. The analyses of total fatty acid compositions of the test oils and faecal lipids were carried out in accordance with earlier work $^{(14)}$. The composition ratio of major NEFA in faeces:individual fatty acids in the test oils was computed to represent the excretion index of each fatty acid. Regiospecific analyses of the test oils and adipose tissue samples were carried out using a JEOL ECA- $400 \mathrm{MHz}$ NMR spectrometer (JEOL Ltd) as reported previously ${ }^{(18)}$. Faecal fatty acids were derivatised, and their absolute amounts were ascertained by GC according to the method of Cheng et al. ${ }^{(19)}$.

\section{Statistical analyses}

All the statistical analyses were carried out using IBM SPSS Statistic 20 (SPSS Inc.). One-way ANOVA was used to assess the significance of differences among the dietary groups, whereas Tukey's honestly significant difference test was used for pairwise comparisons. $P$ values lower than 0.05 were considered significant.

\section{Results}

Food intake, body-mass gain and body-mass gain/g of feed

All the mice exhibited comparable total food intake $(P=0 \cdot 400)$ throughout the experimental period. Food intake on a weekly basis is given in the Supplementary material (available online). Mice fed the HOS-enriched diet gained significantly higher body mass when compared with those fed the COB-enriched $(P=0.030)$ and SAL-enriched $(P<0 \cdot 001)$ diets (Table 3). 
Table 2. Positional fatty acid composition ( $\mathrm{mol} / 100 \mathrm{~mol}$ of total fatty acids) of dietary oils

\begin{tabular}{|c|c|c|c|c|c|}
\hline \multirow[b]{3}{*}{ Dietary oils } & \multicolumn{5}{|c|}{ Composition (mol/100 mol of total fatty acids) } \\
\hline & \multirow[b]{2}{*}{ sn-position } & \multirow[b]{2}{*}{ SFA } & \multicolumn{2}{|c|}{ MUFA } & \multirow[b]{2}{*}{ PUFA } \\
\hline & & & cis-11-Monoene $^{\star}$ & cis-9-Monoene & \\
\hline \multirow[t]{3}{*}{$\mathrm{COB}$} & 1,3 & $94 \cdot 7$ & ND & $5 \cdot 3$ & ND \\
\hline & 2 & $5 \cdot 0$ & ND & 88.9 & $6 \cdot 1$ \\
\hline & $1,2,3$ & 64.9 & ND & $33 \cdot 1$ & $2 \cdot 0$ \\
\hline \multirow[t]{3}{*}{ SAL } & 1,3 & 93.7 & ND & 4.6 & 1.7 \\
\hline & 2 & ND & ND & $100 \cdot 0$ & ND \\
\hline & $1,2,3$ & 62.4 & ND & 36.5 & $1 \cdot 1$ \\
\hline \multirow[t]{3}{*}{ PMF } & 1,3 & $92 \cdot 3$ & ND & 6.5 & $1 \cdot 2$ \\
\hline & 2 & $16 \cdot 4$ & ND & $75 \cdot 9$ & $7 \cdot 7$ \\
\hline & $1,2,3$ & $68 \cdot 3$ & ND & 28.5 & 3.3 \\
\hline \multirow[t]{3}{*}{ HOS } & 1, 3 & $11 \cdot 6$ & $2 \cdot 6$ & 73.9 & 11.9 \\
\hline & 2 & ND & ND & 86.5 & 13.5 \\
\hline & $1,2,3$ & 7.8 & $1 \cdot 7$ & $78 \cdot 1$ & $12 \cdot 4$ \\
\hline
\end{tabular}

Mice fed the SAL-enriched diet also exhibited significantly lower $(P=0.023)$ body-mass gain when compared with those fed the PMF-enriched diet (Table 3). Normalised data indicated significantly lower gain/feed in the COB $(P=0.002)$ and SAL $(P<0 \cdot 001)$ dietary groups than in the HOS dietary group. In addition, mice fed the PMF-enriched diet had significantly higher gain/feed ( $P=0.003)$ compared with those fed the SAL-enriched diet (Table 3 ).

\section{Fat deposition}

Mice in the HOS dietary group exhibited significantly higher subcutaneous fat mass when compared with those in the COB $(P=0.004)$, SAL $(P<0.001)$ and PMF $(P=0.029)$ dietary groups (Table 3 ). Furthermore, the percentage of visceral fat was significantly lower in mice fed the SAL-enriched diet than in those fed the PMF-enriched $(P=0 \cdot 041)$ and
HOS-enriched $(P=0 \cdot 010)$ diets. Overall, the HOS dietary group exhibited significantly higher total fat deposition when compared with the COB $(P=0 \cdot 017)$ and SAL $(P=0 \cdot 001)$ dietary groups.

With respect to the relative amount of total fat contributing to the final body mass, mice in the HOS dietary group exhibited a significantly higher percentage of subcutaneous fat compared with those in the COB $(P=0.008)$, SAL $(P<0.001)$ and PMF $(P=0.036)$ dietary groups. Furthermore, mice in the SAL dietary group displayed a significantly lower percentage of visceral fat $(P=0.035)$ compared with those in the HOS dietary group. With respect to the relative amount of total fat in the carcass, mice fed the HOS-enriched diet exhibited a significantly higher percentage compared with those fed the SAL-enriched $(P=0.002)$ and COB-enriched $(P=0.041)$ diets.

The mass of fat deposited was further subjected to normalisation with total food intake, shorthanded as fat/feed.

Table 3. Effect of dietary oils in C57BL/6 mice after 15 weeks

(Means values with their standard errors)

\begin{tabular}{|c|c|c|c|c|c|c|c|c|c|c|}
\hline & \multicolumn{2}{|c|}{$\mathrm{COB}$} & \multicolumn{2}{|c|}{ SAL } & \multicolumn{2}{|c|}{ PMF } & \multicolumn{2}{|c|}{ HOS } & \multirow[b]{2}{*}{ SED } & \multirow[b]{2}{*}{$P^{\star}$} \\
\hline & Mean & SEM & Mean & SEM & Mean & SEM & Mean & SEM & & \\
\hline Food intake $(\mathrm{g}) \dagger$ & $438 \cdot 15$ & $10 \cdot 22$ & $423 \cdot 18$ & 6.68 & $416 \cdot 78$ & $9 \cdot 19$ & 421.01 & 10.44 & 13.08 & 0.3996 \\
\hline Body-mass gain (g) & $14 \cdot 84^{\mathrm{a}, \mathrm{b}}$ & 0.92 & $12 \cdot 12^{\mathrm{a}}$ & 0.86 & $16 \cdot 77^{\mathrm{b}, \mathrm{c}}$ & 0.99 & $19 \cdot 33^{c}$ & 1.48 & 1.54 & 0.0004 \\
\hline Subcutaneous fat $(\mathrm{g})$ & $1.05^{\mathrm{a}}$ & 0.13 & $0.76^{a}$ & 0.14 & $1 \cdot 22^{\mathrm{a}}$ & 0.18 & $1.87^{\mathrm{b}}$ & 0.18 & 0.22 & 0.0001 \\
\hline Visceral fat $(\mathrm{g})$ & $1.56^{\mathrm{a}, \mathrm{b}}$ & $0 \cdot 18$ & $1 \cdot 29^{a}$ & 0.22 & $2 \cdot 15^{\mathrm{b}}$ & 0.26 & $2 \cdot 32^{b}$ & 0.21 & 0.31 & 0.0056 \\
\hline Total fat $(\mathrm{g})$ & $2 \cdot 60^{\mathrm{a}}$ & 0.30 & $2 \cdot 04^{\mathrm{a}}$ & 0.35 & $3.37^{\mathrm{a}, \mathrm{b}}$ & 0.41 & $4 \cdot 20^{b}$ & 0.37 & 0.51 & 0.0009 \\
\hline \multicolumn{11}{|l|}{ Fat percentage in the final body mass } \\
\hline Percentage of subcutaneous fat & $2 \cdot 98^{\mathrm{a}}$ & 0.32 & $2 \cdot 27^{\mathrm{a}}$ & 0.35 & $3.29^{\mathrm{a}}$ & 0.44 & $4.73^{\mathrm{b}}$ & 0.31 & 0.51 & 0.0003 \\
\hline Percentage of visceral fat & $4.46^{\mathrm{a}, \mathrm{b}}$ & 0.44 & $3.87^{\mathrm{a}}$ & 0.52 & $5 \cdot 76^{a, b}$ & 0.63 & $5 \cdot 92^{b}$ & 0.42 & 0.72 & 0.0179 \\
\hline Percentage of total fat & $7 \cdot 45^{\mathrm{a}}$ & 0.71 & $6 \cdot 14^{\mathrm{a}}$ & 0.85 & $9 \cdot 05^{\mathrm{a}, \mathrm{b}}$ & 0.98 & $10 \cdot 65^{\mathrm{b}}$ & 0.68 & $1 \cdot 15$ & 0.0025 \\
\hline \multicolumn{11}{|l|}{ Normalised with food intake } \\
\hline Body-mass gain/feed $\left(\times 10^{-2} \mathrm{~g} / \mathrm{g}\right)$ & $3.37^{\mathrm{a}, \mathrm{b}}$ & 0.16 & $2 \cdot 86^{\mathrm{a}}$ & 0.19 & $4 \cdot 02^{\mathrm{b}, \mathrm{c}}$ & 0.22 & $4 \cdot 55^{c}$ & 0.27 & 0.30 & $<0.0001$ \\
\hline Subcutaneous fat/feed $\left(\times 10^{-3} \mathrm{~g} / \mathrm{g}\right)$ & $2 \cdot 4^{\mathrm{a}}$ & 0.3 & $1 \cdot 8^{\mathrm{a}}$ & 0.3 & $2 \cdot 9^{a}$ & 0.4 & $4 \cdot 4^{\mathrm{b}}$ & 0.4 & 0.48 & $<0.0001$ \\
\hline Visceral fat/feed $\left(\times 10^{-3} \mathrm{~g} / \mathrm{g}\right)$ & $3 \cdot 6^{\mathrm{a}, \mathrm{b}}$ & 0.4 & $3 \cdot 0^{\mathrm{a}}$ & 0.5 & $5 \cdot 1^{\mathrm{b}, \mathrm{c}}$ & 0.6 & $5 \cdot 5^{\mathrm{c}}$ & 0.5 & 0.70 & 0.0028 \\
\hline Total fat $/ \mathrm{feed}\left(\times 10^{-3} \mathrm{~g} / \mathrm{g}\right)$ & $5 \cdot 9^{\mathrm{a}, \mathrm{b}}$ & 0.7 & $4 \cdot 8^{\mathrm{a}}$ & 0.8 & $8 \cdot 0^{\mathrm{b}, \mathrm{c}}$ & 0.9 & $9 \cdot 9^{c}$ & 0.8 & $1 \cdot 12$ & 0.0003 \\
\hline
\end{tabular}

$\mathrm{COB}$, cocoa butter; SAL, sal stearin; PMF, palm mid fraction; HOS, high-oleic sunflower oil.

a,b,c Mean values within a row with unlike superscript letters were significantly different $(P<0.05)$.

${ }^{*} P$ values were determined by one-way ANOVA.

†Food intake was calculated as the total food intake for each mouse in 15 weeks and then averaged for each group ( $n$ 10). 
Subcutaneous fat/feed (Table 3) of the HOS dietary group was significantly higher than that of the COB $(P=0 \cdot 001)$, SAL $(P<0.001)$ and PMF $(P=0.016)$ dietary groups. The values of visceral fat/feed and total fat/feed were significantly lower in the SAL $(P=0.007$ and $P<0.001$, respectively) and COB ( $P=0.043$ and $P=0.006$, respectively) dietary groups than in the HOS dietary group. Furthermore, mice fed the PMF-enriched diet continued to exhibit significantly higher visceral fat/feed $(P=0.029)$ compared with those fed the SAL-enriched diet (Table 3 ). It is worth noting that significantly lower amounts $(P=0.037)$ of total fat deposited per $g$ of feed consumed were observed in the SAL dietary group than in the PMF dietary group. On the other hand, the COB dietary group exhibited non-significant differences for all the parameters when compared with both the SAL and PMF dietary groups.

As shown in Fig. 1, a poor correlation was observed between feed efficiency (fat/feed) and total SFA content at $s n-1,3$ positions of TAG in dietary fats $(r$ 0.6847-0.9195). Correlation coefficients for all the parameters were nonsignificant, as the resultant $P$ values were greater than 0.05 . Nevertheless, when fat/feed was plotted against stearic acid content at $s n-1,3$ positions, negative linear correlations were observed for subcutaneous fat/feed, visceral fat/feed and total fat/feed ( $r$ 0.9693, 0.9989 and 0.9999, respectively) (Fig. 2). Correlation coefficients for both total fat/feed and visceral fat/feed against stearic acid content at $s n-1,3$ positions were found to be statistically significant at $P$ values of $0 \cdot 011$ and 0.030 , respectively.

\section{Faecal fatty acids}

The lipid fraction of mouse faeces mainly contained NEFA, while TAG and other partial acylglycerols remained undetected. The composition of faecal NEFA of each dietary group is given in the Supplementary material (available online). With respect to the absolute amount of faecal fatty acids excreted (Table 4), the SAL dietary group excreted significantly higher amounts of NEFA than the COB $(P=0.038)$, PMF $(P=0.031)$ and HOS $(P=0.002)$ dietary groups. Among the four dietary groups, the HOS dietary group excreted the lowest amounts of faecal NEFA (Table 4).

The excretion index denotes the relationship between faecal fatty acids and their corresponding composition in the ingested fats (Table 5). The most abundant MUFA, namely oleic acid, is most readily absorbed, as the excretion indices fall in the range of $0 \cdot 28-0.60$. In other words, the relative composition of oleic acid in faeces is lower than that in dietary fats. On the contrary, the major SFA, namely palmitic and stearic acids, are relatively more readily excreted, as their excretion indices are greater than unity. Greater excretion indices were observed for stearic acid (1.32-3.87) than for palmitic acid $(1 \cdot 15-3 \cdot 33)$ in all the dietary groups (Table 5).

\section{Discussion}

In the present study, $\mathrm{C} 57 \mathrm{BL} / 6$ mice were employed as this strain has been reported to be a robust and efficient animal model for human obesity and type 2 diabetes as well as to remain lean if the fat content of the diet is limited ${ }^{(20-22)}$. Most of the animal models do not fulfil these requirements, as they are mainly based on monogenic disorders of limited relevance to human obesity and diabetes. Moreover, C57BL/6 mice exhibit intact leptin signalling and display delayed-onset obesity that can be amplified in response to high-fat diets, making them an ideal model for human obesity studies $^{(23)}$. In addition, this mouse model has a genetic factor similar to that of humans that causes diabetes after the development of obesity ${ }^{(20)}$.

The major TAG present in the test oils, namely COB, SAL, PMF and HOS, used in the present study were 1-palmitoyl2-oleoyl-3-stearoyl glycerol (POSt), 1,3-distearoyl-2-oleoyl

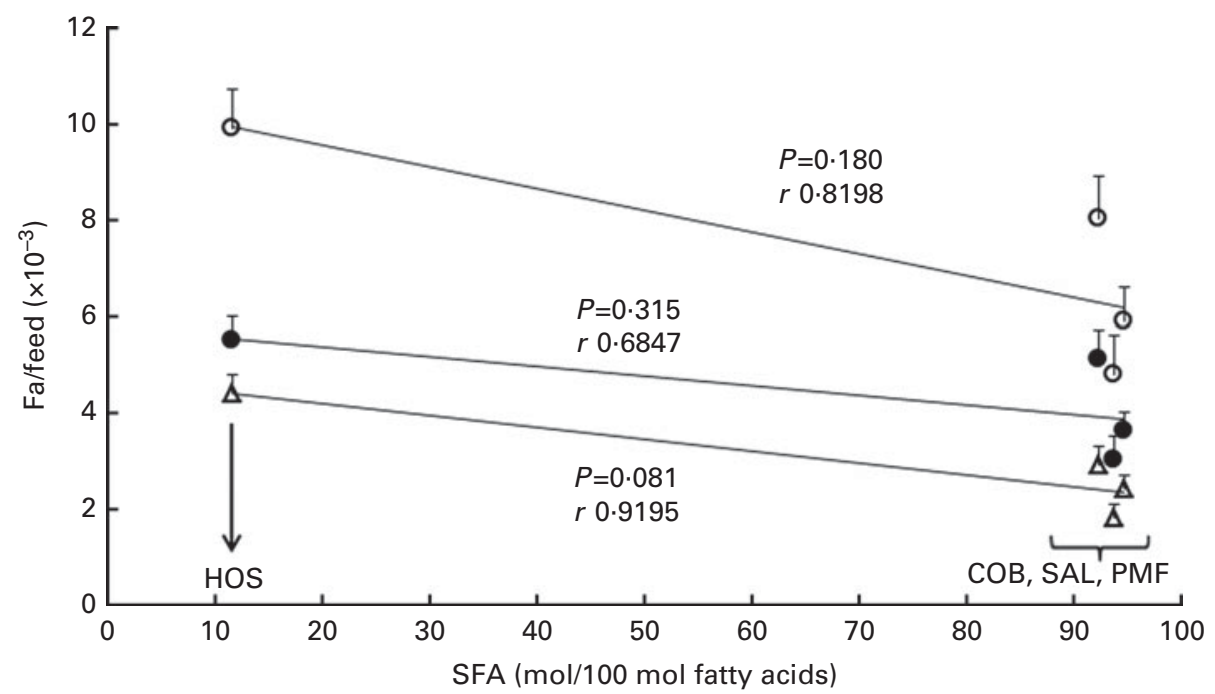

Fig. 1. Effect of SFA content at $s n-1,3$ positions of TAG on fat deposition (mass of fat deposited/total feed consumed). Values are means for each group ( $n$ 10), with their standard errors represented by vertical bars. Correlation coefficients with $P$ values $<0.05$ were considered significant. COB, cocoa butter; SAL, sal stearin; PMF, palm mid fraction; HOS, high-oleic sunflower oil; $r$, correlation coefficients. $\bigcirc$, Total fat/feed; $\Delta$, subcutaneous fat/feed; $\bullet$, visceral fat/feed. 


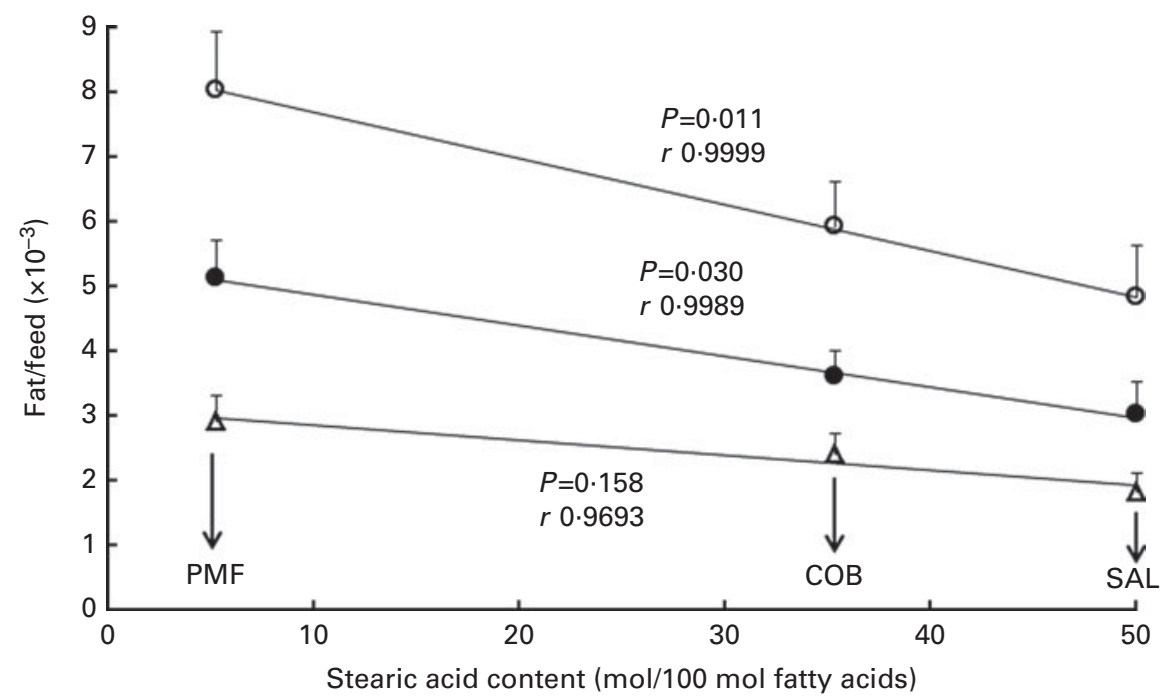

Fig. 2. Effect of stearic acid content at sn-1, 3 positions of TAG on fat deposition (mass of fat deposited/total feed consumed). Values are means for each group ( $n$ 10), with their standard errors represented by vertical bars. Correlation coefficients with $P$ values $<0.05$ were considered significant. COB, cocoa butter; $\mathrm{SAL}$, sal stearin; PMF, palm mid fraction; $r$, correlation coefficients. Data obtained for the high-oleic sunflower oil dietary group were not included, as the factor affecting fat deposition was the high MUFA content at $s n-1,3$ positions. $\bigcirc$, Total fat/feed; $\Delta$, subcutaneous fat/feed; $\bullet$, visceral fat/feed.

glycerol (StOSt), 1,3-dipalmitoyl-2-oleoylglycerol and trioleoylglycerol, respectively ${ }^{(24)}$. In an effort to examine the obesity-alleviating effect of SFA at $s n-1,3$ positions, these positions were varied among palmitic, stearic and oleic acids, whilst $s n-2$ position was mainly conserved with oleic acid (Table 2). In addition, normalised data, namely gain/feed and fat/feed, were analysed and subcutaneous adiposity and visceral adiposity were used as markers of fat deposition.

\section{Long-chain SFA at sn-1, 3 positions reduce fat deposition}

Throughout the 15 weeks of the experimental period, there was no discrepancy in the food intake of all the dietary groups, suggesting that all the test oils exerted similar effects on hunger and appetite. In line with previous findings ${ }^{(14)}$, the diet enriched with HOS (MUFA mainly present at $s n-1,3$ positions) was found to cause higher fat deposition than diets with long-chain SFA at similar positions, as observed in the COB, SAL and PMF dietary groups. Furthermore, significantly greater amounts of faecal fatty acids were observed in the latter groups (Table 4), suggesting that the greater excretion of long-chain SFA at $s n-1,3$ positions contributed to the lower fat deposition observed. The amount of resynthesised TAG was reduced, affecting their participation in subsequent metabolism and their distribution in tissues and eventually the extent of fat deposition. Therefore, this further validates our postulation, i.e. when long-chain SFA are present at $s n-1$ and $s n-3$ positions, the extent of fat deposition will be alleviated. In contrast, the major MUFA, namely oleic acid, was found to be least readily excreted (excretion indices $<1$; Table 5 ), as it was well absorbed in the intestine.

\section{Stearic acid at sn-1, 3 positions is more efficient than palmitic acid at sn-1, 3 positions at reducing fat deposition}

Surprisingly, SAL had been shown to lead to significantly lower gain in body mass as well as visceral and total fat mass compared with PMF, despite their similarity in the positional distribution of long-chain SFA. This observation was concomitant with the significantly higher amounts of faecal

Table 4. Absolute amount of NEFA in the faeces of mice of different dietary groups

(Mean values and standard deviations)

\begin{tabular}{|c|c|c|c|c|c|c|c|c|}
\hline \multirow[b]{3}{*}{ Fatty acids } & \multicolumn{8}{|c|}{ Absolute amount of fatty acids ( $\mu \mathrm{g} / 1 \mathrm{~g}$ of faeces) } \\
\hline & \multicolumn{2}{|c|}{$\mathrm{COB}$} & \multicolumn{2}{|c|}{ SAL } & \multicolumn{2}{|c|}{ PMF } & \multicolumn{2}{|c|}{ HOS } \\
\hline & Mean & SD & Mean & SD & Mean & SD & Mean & SD \\
\hline $16: 0$ & $2 \cdot 39^{a}$ & $0 \cdot 16$ & $0.53^{b}$ & 0.04 & $5 \cdot 06^{c}$ & 0.31 & $0.55^{b}$ & $0 \cdot 10$ \\
\hline $18: 0$ & $4 \cdot 00^{\mathrm{a}}$ & 0.20 & $8 \cdot 02^{b}$ & 0.93 & $0.91^{c}$ & 0.20 & $0.67^{c}$ & 0.12 \\
\hline $18: 1 n-9$ & $1.98^{\mathrm{a}}$ & 0.41 & $1.63^{\mathrm{a}}$ & 0.41 & $1 \cdot 87^{\mathrm{a}}$ & 0.91 & $3.50^{b}$ & 0.60 \\
\hline $18: 2 n-6$ & $0.39^{a}$ & 0.10 & $0 \cdot 69^{a}$ & 0.15 & $0.44^{a}$ & 0.18 & $1 \cdot 45^{b}$ & 0.08 \\
\hline $20: 0$ & $0.88^{a}$ & 0.07 & $1.55^{\mathrm{b}}$ & 0.23 & $0.09^{c}$ & 0.04 & $0 \cdot 15^{c}$ & 0.03 \\
\hline Total fatty acids & $9 \cdot 64^{a}$ & 0.78 & $12 \cdot 42^{b}$ & 1.01 & $8 \cdot 36^{a}$ & 0.93 & $6 \cdot 31^{c}$ & 0.55 \\
\hline
\end{tabular}

COB, cocoa butter; SAL, sal stearin; PMF, palm mid fraction; HOS, high-oleic sunflower oil.

${ }^{\mathrm{a}, \mathrm{b}, \mathrm{c}}$ Mean values of total fatty acids for each dietary group $(n 10)$ with unlike superscript letters were significantly different $(P<0.05)$. 
Table 5. Excretion indices for major fatty acids in the faeces of mice of different dietary groups

\begin{tabular}{|c|c|c|c|c|}
\hline \multirow[b]{2}{*}{ Fatty acids } & \multicolumn{4}{|c|}{ Excretion index } \\
\hline & $\mathrm{COB}$ & SAL & PMF & HOS \\
\hline $16: 0$ & 1.24 & 1.19 & $1 \cdot 15$ & 3.33 \\
\hline $18: 0$ & 1.32 & 1.33 & 1.86 & 3.87 \\
\hline $18: 1 n-9$ & 0.32 & 0.28 & 0.39 & 0.60 \\
\hline
\end{tabular}

NEFA excreted by the SAL dietary group (Table 4), as well as the relatively higher excretion index of stearic acid in comparison with that of palmitic acid (Table 5). In line with the previous literature ${ }^{(25)}$, the extent of SFA excretion is related to the chain length, i.e. stearic acid is more readily excreted than palmitic acid. Consequently, this phenomenon has been shown to exert a remarkable impact on fat deposition.

When normalised data (fat/feed) were plotted against total SFA content at $s n-1,3$ positions (Fig. 1), the linear correlation as suggested previously ${ }^{(14)}$ was not observed in the present study. Palmitic acid at $s n-1,3$ positions, which was the sole major SFA, was responsible for the notable linear correlation observed in the previous study ${ }^{(14)}$, while in the present study, varying amounts of stearic and palmitic acids at $s n-1,3$ positions were employed for comparison. Therefore, a negative linear correlation with exceptional correlation coefficients was only observed when feed efficiency (fat/feed) was correlated with the amount of individual stearic acids at $s n-1,3$ positions (Fig. 2). This suggests that both stearic and palmitic acids exert differential effects on fat absorption and subsequently fat deposition. Data obtained for the HOS dietary group are not shown in Fig. 2, as the factor affecting fat deposition was the high MUFA content at $s n-1,3$ positions. Besides the fatreducing effect of long-chain SFA at $s n-1,3$ positions proposed previously $^{(14)}$, an extended finding is that stearic acid is more efficient at limiting fat deposition than palmitic acid at similar positions.

For efficient intestinal absorption, micelles formed by bile salts transport the non-polar stearic and palmitic acids across the aqueous boundary layer of intestinal wall. The equilibrium between non-polar and aqueous phases and the rate at which the NEFA establish equilibrium between the two phases are crucial for determining the absorption of fatty acids ${ }^{(26)}$. Poor diffusion of fatty acids into the aqueous phase may inhibit the action of enzymes by blocking the interface. As stearic acid is relatively more apolar than palmitic acid, a lower trend towards intestinal absorption is expected. This has been demonstrated by the remarkably higher amounts of excreted NEFA in the faeces of the SAL dietary group than in that of the PMF dietary group (Table 4). The reduced absorption of stearic acid will subsequently lead to the reduction of resynthesised TAG levels in chylomicron and their distribution in tissues and eventually the extent of fat deposition.
Furthermore, differences in the physio-chemical properties of Ca soaps of fatty acids may give noteworthy insights into their different extent of faecal excretion. For instance, Krafft point for a Ca soap of oleic acid is $31^{\circ} \mathrm{C}$, for that of palmitic acid is $65^{\circ} \mathrm{C}$ and for that of stearic acid is $82^{\circ} \mathrm{C}^{(27)}$. Calcium palmitate and calcium stearate are thus solid at body temperature. Consequently, there is no value for the critical micelle concentration as micelles cannot be formed. In general, Krafft points of all the fatty acids are elevated in the presence of Ca ions ${ }^{(27)}$. Furthermore, the solubility of Ca salt of palmitic acid in water has been reported to be $0.012 \mathrm{mg} / \mathrm{l}$, while a lower extent of dissolution, i.e. $0.0007 \mathrm{mg} / \mathrm{l}$, has been observed for calcium stearate at $25^{\circ} \mathrm{C}(28)$. Hence, the intestinal absorption of NEFA probably appears to be determined by both hydrophobicity and the Krafft point in aqueous solution. In addition to malabsorption-type effects, the reduced feed efficiency and adiposity observed in the present study may also have occurred as a result of increasing fat oxidation and energy expenditure.

Regiospecific analysis of adipose tissue samples (see Supplementary material, available online) indicated a tremendous depreciation of SFA at $s n-1,3$ positions (44.1-64.9 mol\%), suggesting the lower intestinal absorption of SFA and de novo synthesis by desaturation. In addition, mead acid (20:3n-9), which is an indicator for the deficiency of essential fatty acids, was not detected in the subcutaneous as well as visceral adipose tissue samples of mice of all the dietary groups (see Supplementary material, available online). The present study demonstrates that fats predominantly comprised of high amounts of StOSt and POSt, namely SAL and COB, respectively, are associated with lower visceral adiposity. This finding is crucial as visceral adiposity is associated with various diseases, including CVD, insulin resistance, hypertension and overall mortality ${ }^{(29)}$. Hence, shea butter and mango fat, which contain comparable major TAG species, are expected to exert similar favourable health effects ${ }^{(24)}$.

The observation reported in previous literature ${ }^{(4)}$ that $\mathrm{COB}$ is better at preventing fat accretion than palm oil has been successfully elucidated in the present study. Besides this, in our previous work $^{(14)}$, we observed that dietary oil containing high amounts of linoleic and linolenic acids at $s n-1,3$ positions, such as soyabean oil, led to significantly higher fat deposition than the diet enriched with palm olein. With the relatively higher trend exhibited by MUFA and PUFA towards intestinal absorption, diets that contain low amounts of the above-mentioned fatty acids, especially at the $s n-1,3$ positions, are expected to lead to a lower risk of body fat deposition. Therefore, we postulate that COB with StOSt and POSt as the major TAG species compared with various food products exhibits a lower tendency to cause obesity and visceral adiposity when compared with highly unsaturated oil products. In conclusion, the encouraging results of the present study may warrant further investigation in human subjects, as well as the synthesis of prospective healthier structured lipids, for instance, 1,3-dibehenoyl-2-oleoylglycerol and 1,3-dilignoceroyl2-oleoylglycerol, which have the potential to reduce the prevalence of morbid obesity. 


\section{Supplementary material}

To view supplementary material for this article, please visit http://dx.doi.org/10.1017/S0007114513003668

\section{Acknowledgements}

The present study was funded by the Malaysian Ministry of Higher Education (Fundamental Research Grant Scheme) and University of Malaya (Postgraduate Research Fund) through grants FP011/2010A and PV017/2011B, respectively. The Malaysian Ministry of Higher Education and University of Malaya had no role in the design and analysis of the study or in the writing of this article.

The authors' contributions are as follows: S.-W. G. carried out the hands-on experiments, namely the animal study, sample analyses and statistical analyses; S.-W. G. and S.-F. C. wrote the manuscript; A. S.-H. O. and C.-H. C. read and approved the final version. All the authors were responsible for the design and execution of the study.

None of the authors has any conflicts of interest to declare.

\section{References}

1. Sanz M, Lopez-Bote CJ, Menoyo D, et al. (2000) Abdominal fat deposition and fatty acid synthesis are lower and $\beta$-oxidation is higher in broiler chickens fed diets containing unsaturated rather than saturated fat. J Nutr 130, 3034-3037.

2. Hariri N, Gougeon R \& Thibault L (2010) A highly saturated fat-rich diet is more obesogenic than diets with lower saturated fat content. Nutr Res 30, 632-643.

3. Matsuo T, Takeuchi H, Suzuki H, et al. (2002) Body fat accumulation is greater in rats fed a beef tallow diet than in rats fed a safflower or soybean oil diet. Asia Pacific J Clin Nutr 11, 302-308.

4. Timmers S, de Vogel-van den Bosch J, de Wit N, et al. (2011) Differential effects of saturated versus unsaturated dietary fatty acids on weight gain and myocellular lipid profiles in mice. Nutr Diabetes 1, e11.

5. Stachon M, Furstenberg E \& Gromadzka-Ostrowska J (2006) Effects of high-fat diets on body composition, hypothalamus NPY, and plasma leptin and corticosterone levels in rats. Endocrine 30, 69-74.

6. Jen KL, Buison A, Pellizzon M, et al. (2003) Differential effects of fatty acids and exercise on body weight regulation and metabolism in female Wistar rats. Exp Biol Med $\mathbf{2 2 8}$, 843-849

7. Ikemoto S, Takahashi M, Tsunoda N, et al. (1996) High-fat diet-induced hyperglycemia and obesity in mice: differential effects of dietary oils. Metabolism 45, 1539-1546.

8. Newman RE, Bryden WL, Fleck E, et al. (2002) Dietary $n$-3 and $n-6$ fatty acids alter avian metabolism: metabolism and abdominal fat deposition. Br J Nutr 88, 11-18.

9. Czernichow S, Thomas D \& Bruckert E (2010) n-6 Fatty acids and cardiovascular health: a review of the evidence for dietary intake recommendations. Br J Nutr 104, 788-796.

10. Okuno M, Kajiwara K, Imai S, et al. (1997) Perilla oil prevents the excessive growth of visceral adipose tissue in rats by down-regulating adipocyte differentiation. $J \mathrm{Nutr}$ 127, 1752-1757.
11. Micallef M, Munro I, Phang M, et al. (2009) Plasma $n-3$ polyunsaturated fatty acids are negatively associated with obesity. Br J Nutr 102, 1370-1374.

12. Martinez-Victoria E \& Yago MD (2012) Omega 3 polyunsaturated fatty acids and body weight. Br J Nutr 107, S107-S116.

13. Kojima M, Tachibana N, Yamahira T, et al. (2010) Structured triacylglycerol containing behenic and oleic acids suppresses triacylglycerol absorption and prevents obesity in rats. Lipids Health Dis 9, 77-82.

14. Gouk SW, Cheng SF, Mok JSL, et al. (2013) Long-chain SFA at the $s n-1,3$ positions of TAG reduce body fat deposition in C57BL/6 mice. Br J Nutr (epublication ahead of print 12 June 2013).

15. Mattson FH, Nolen GA \& Webb MR (1979) The absorbability by rats of various triglycerides of stearic and oleic acid and the effect of dietary calcium and magnesium. J Nutr 109, $1682-1687$.

16. Brink EJ, Haddeman E, de Fouw NJ, et al. (1995) Positional distribution of stearic acid and oleic acid in a triacylglycerol and dietary calcium concentration determines the apparent absorption of these fatty acids in rats. J Nutr 125, 2379-2387.

17. Folch J, Leens M \& Sloane-Stanley GH (1957) A simple method for the isolation and purification of total lipids from animal tissue. J Biol Chem 226, 497-509.

18. Gouk SW, Cheng SF, Ong ASH, et al. (2012) Rapid and direct quantitative analysis of positional fatty acids in triacylglycerols using ${ }^{13} \mathrm{C}$ NMR. Eur J Lipid Sci Technol 114, 510-519.

19. Cheng SF, Choo YM, Ma AN, et al. (2005) Rapid synthesis of palm-based monoacylglycerols. J Am Oil Chem Soc 82, 791-795.

20. Surwit RS, Kuhn CM, Cochrane C, et al. (1988) Diet-induced type II diabetes in C57BL/6J mice. Diabetes 37, 1163-1167.

21. Surwit RS, Feinglos MN, Rodin J, et al. (1995) Differential effects of fat and sucrose on the development of obesity and diabetes in $\mathrm{C} 57 \mathrm{BL} / 6 \mathrm{~J}$ and $\mathrm{A} / \mathrm{J}$ mice. Metabolism $\mathbf{4 4}$, 645-651.

22. Winzell MS \& Ahrén B (2004) The high-fat diet-fed mouse: a model for studying mechanisms and treatment of impaired glucose tolerance and type 2 diabetes. Diabetes $\mathbf{5 3}$, S215-S219.

23. Kennedy AJ, Ellacott KLJ, King VL, et al. (2010) Mouse models of the metabolic syndrome. Dis Model Mech 3, $156-166$.

24. Gunstone FD \& Harwood JL (2007) Occurrence and characterisation of oils and fats. In The Lipid Handbook, 3rd ed., pp. 92-108 [FD Gunstone, JL Harwood and AJ Dijkstra, editors]. Boca Raton, FL: CRC Press.

25. Lien EL, Yuhas RJ, Boyle FG, et al. (1993) Corandomization of fats improves absorption in rats. J Nutr 123, 1859-1867.

26. Freeman CP (1969) Properties of fatty acids in dispersion of emulsified lipid and bile salt and the significance of these properties in fat absorption in the pig and the sheep. $\mathrm{BrJ}$ Nutr 23, 249-263.

27. Yamaguchi T, Kaneda M \& Kakinuma K (1986) Effect of saturated and unsaturated fatty acids on the oxidative metabolism of human neutrophils. The role of calcium ion in the extracellular medium. Biochim Biophys Acta 861, 440-446.

28. Irani RR \& Callis CF (1960) Metal complexing by phosphorus compounds II. Solubility of calcium soaps of linear carboxylic acids. J Phys Chem 64, 1741-1743.

29. Emery EM, Schmid TL, Kahn HS, et al. (1993) A review of the association between abdominal fat distribution, health outcome measures, and modifiable risk factors. Am J Health Promot 7, 342-353. 